\title{
Fonction épistémique de l'écrit et genres disciplinaires Enquête dans les classes d'histoire et de sciences du secondaire québécois
}

\section{Suzanne-G. Chartrand, Christiane Blaser et Mathieu Gagnon}

Les réformes des curriculums scolaires reconnaissent la place fondamentale du langage dans la construction des connaissances par les élèves. Quel rôle joue l'écrit (ce qui est lu et écrit) dans les classes d'histoire et de sciences du secondaire? Pour répondre à cette question, nous menons une enquête sur les pratiques de lecture et d'écriture dans ces disciplines. Après avoir présenté le cadre conceptuel et la méthodologie d'enquête par questionnaire, nous exposerons les premiers résultats sur le rapport à l'écrit d'elèves du secondaire au Québec.

Au cours des dernières années, plusieurs systèmes éducatifs de pays occidentaux ont mené des réformes des curriculums ${ }^{1}$ scolaires. Dans les pays francophones, notamment, les autorités scolaires, reconnaissant l'importance du langage dans la construction des savoirs, considèrent que développer les compétences langagières des élèves relève de la responsabilité de tous les intervenants scolaires, pas seulement des enseignants de français. Au Québec, le ministère de l'Éducation a fait de la langue une "compétence transversale». Bien que de nouvelles pratiques soient nécessaires pour que soit développée cette «compétence» dans toutes les disciplines, les données sur l'actuelle prise en charge de cette dimension cruciale de l'apprentissage dans les disciplines autres que le français font défaut. Nous avons donc entrepris une recherche descriptive sur les pratiques de lecture et d'écriture dans les cours d'histoire et de sciences du secondaire au Québec (élèves de 12 à 17 ans).

Dans la première partie de cet article, nous exposerons brièvement les fondements de la recherche en cours ${ }^{2}$. Suivra, en deuxième partie, une présentation de la démarche méthodologique d'élaboration et de validation du questionnaire soumis aux élèves. Dans la troisième partie, nous présenterons les résultats à deux questions du questionnaire élèves à propos de la fonction épistémique de l'écriture et des genres scolaires produits par les élèves dans les deux disciplines choisies. Nous conclurons en soulignant l'apport possible de cette étude à différents champs de recherches en sciences de l'éducation. 


\section{Les fondements théoriques de la recherche}

Précisons d'emblée que cette étude se situe dans un nouveau champ de recherches en didactique du français qui prend comme cadre théorique l'interactionnisme social d'inspiration vygotskienne s'intéressant plus spécifiquement aux rapports entre pensée, conceptualisation, langage écrit et apprentissage scolaire (Vygotski, 1997/1934). Ce champ s'inscrit dans la mouvance de l'interactionnisme sociodiscursif théorisé et opérationnalisé par l'équipe de didactique des langues de l'Université de Genève sous la direction de J.-P. Bronckart (Bronckart, Bain, Schneuwly, Davaud \& Pasquier, 1985; Bronckart, 1996). Trois thèmes de recherche constituent l'armature de la présente étude: le rapport à l'écrit, la fonction épistémique de l'écrit et le genre textuel disciplinaire (genre de texte propre à une discipline scolaire).

\section{Le rapport à l'écrit}

La compréhension des activités scolaires menées en lecture et en écriture passe, entre autres, par l'étude du rapport à l'écrit des enseignants et des élèves. Pour explorer ce rapport, nous nous sommes inspirés du concept de rapport à l'écriture élaboré par Barré-De Miniac (2002). Sommairement, nous définissons le rapport à l'écrit comme l'ensemble des significations construites par un sujet à propos de l'écrit (la lecture et l'écriture). Pour rendre opérationnel ce concept, nous distinguons quatre dimensions dans ce rapport: affective, axiologique, cognitive et praxéologique. La dimension affective concerne les sentiments et les émotions entourant l'écrit ainsi que l'attachement émotif à l'écrit. Elle se manifeste par deux indicateurs: le temps et la fréquence des pratiques de lecture et d'écriture à l'école et hors de l'école ainsi que les efforts que le sujet consacre à l'écrit pour l'école: efforts pour corriger son texte, pour bien le présenter, pour s'assurer qu'il comprend bien ce qu'il lit... La dimension axiologique concerne les valeurs que le sujet accorde à l'écriture et à la lecture pour vivre et s'épanouir au sein d'une société ayant un haut niveau de littératie et pour réussir à l'école comme dans chaque discipline. La dimension cognitive renvoie aux idées, aux représentations ou aux conceptions que se fait le sujet de la place de l'écrit dans la société, de sa ou de ses fonctions dans les apprentissages scolaires en général et dans chaque discipline scolaire en particulier. Enfin, les principaux éléments de la dimension praxéologique ont trait aux activités concrètes, observables des sujets en matière d'écriture et de lecture: ce qu'ils lisent et écrivent, quand et comment ils mènent ces activités.

Concrètement, nous voulons savoir et comprendre quel intérêt affectif les élèves vouent à l'écrit, quelles valeurs ils lui associent, quelles conceptions ils ont de son rôle dans la société, à l'école et dans les disciplines concernées par l'enquête, et quelles sont leurs pratiques effectives.

\section{La fonction épistémique du langage écrit}

La transmission, l'appropriation et la coconstruction ${ }^{3}$ des connaissances font ap- 
pel à des capacités cognitives de haut niveau, en particulier à des capacités langagières de lecture et d'écriture, de là notre intérêt pour la fonction épistémique de l'écrit que nous définissons comme le rôle que joue l'écrit dans l'appropriation et la coconstruction de connaissances et d'habiletés disciplinaires. Barré-De Miniac (1995) et Reuter (2001) reconnaissent que la didactique du français a mis du temps à s'intéresser à la fonction cognitive de l'écriture 4 , "notion extrapolée des travaux de Jack Goody [1976]». Barré-De Miniac (op. cit.) résume ainsi l'apport de l'anthropologue britannique: l'avènement de «l'ordre scriptural» (Peytard, 1970) rend possible des modes de pensée rationnels abstraits et scientifiques engendrant des possibilités cognitives nouvelles au plan du développement de l'espèce humaine (phylogénétique) et, par conséquent, du développement de l'individu (ontogénétique).

Pour réussir des études secondaires, une certaine maitrise de l'écrit normé est nécessaire. Or, on sait que savoir écrire ne se limite pas à appliquer convenablement un ensemble de règles et de normes propres à la langue écrite, souvent assimilée à tort à un «code», mais implique aussi, entre autres, que le sujet scripteur se soit construit des représentations adéquates de différents genres d'écrits, en particulier d'écrits scolaires dans le cas d'élèves (Chartrand, 2000). C'est dire que le scripteur doit avoir accédé à l'ordre scriptural, différent de l'ordre oral.

En effet, une certaine maitrise de l'écrit, notamment dans le cadre des apprentissages scolaires, fait intervenir des instances psychologiques de gestion et de contrôle de la production langagière, lesquelles sont différentes de celles de l'oral à cause des exigences formelles plus grandes de l'écrit et, surtout, de sa dimension plus abstraite (Schneuwly, 1988). Privé d'interactions et généralement obligé de gérer seul sa production langagière, le scripteur doit effectuer un travail cognitif plus complexe. Par exemple, il doit se construire une représentation de plusieurs des composantes de la situation de communication (destinataire, lieu et moment de réception) qui, à l'oral, sont des données sensibles facilitant la production du discours (Vygotski, 1997/1934, 340 et ss.). Ainsi l'écrit scolaire requiert-il des capacités cognitives d'abstraction et d'anticipation plus élaborées que l'oral, lequel, de plus, à l'école comme dans la société, bénéficie d'une plus grande tolérance par rapport à la norme et d'une gamme variationnelle plus vaste.

Dans le monde anglo-saxon, la reconnaissance du rôle du langage dans le processus de construction des connaissances en contexte scolaire a alimenté un important champ de recherches depuis plus de vingt-cinq ans (Simard, 2000), dont le mouvement Writing across the Curriculum (Halliday \& Martin, 1993; Langer \& Applebee, 1987; Sutton, 1995) et Language for academic Purposes (Commander \& Smith, 1996; Mc Crindle \& Christensen, 1995). Sous l'influence de ces mouvements, plusieurs pratiques d'apprentissage au moyen d'activités d'écriture ont été expérimentées à tous les ordres d'enseignement aux États-Unis et en Australie, dans différentes disciplines (Ackerman, 1993; Rowel, 19975). Ce courant de recherches met en lumière l'importance de travailler les spécificités des écrits disciplinaires. 
Récemment, en didactique du français, un autre courant de recherches s'est constitué autour de «l'écrit pour penser et apprendre» (Barré-De Miniac \& Reuter, 2000; Chabanne \& Bucheton, 2002; Delcambre, Dolz \& Simard, 2000) et autour du concept de communauté discursive (Bernié, 2004). Étant donné que le langage est indissociable de l'interaction sociale dans et par laquelle il se réalise, il possède des particularités dans chaque discipline scientifique comme dans chaque discipline scolaire: on ne pense, ni ne parle, ni n'écrit en mathématiques comme en littérature ou en biologie, d'où l'idée de communauté discursive propre à chaque discipline scientifique et, dans une certaine mesure, à chaque discipline scolaire.

\section{Les genres disciplinaires}

Lactivité langagière au cœur des disciplines scolaires s'inscrit donc dans des cadres sociodiscursifs propres aux différentes disciplines, ce qui implique la prise en compte des genres propres à chaque discipline. Dans le modèle de production langagière développé sous la direction de Bronckart (Bronckart, Bain, Schneuwly, Davaud \& Pasquier 1985; Bronckart, 1996), lire et écrire, c'est agir langagièrement au moyen d'outils sémiotiques dont les genres sont les «méga-outils» (Schneuwly, 1995, p. 78). L'activité langagière de l'ordre scriptural se développe par l'élaboration de genres «seconds» (Bakhtine, 1984), qui sont des formes discursives historiquement construites, relativement stabilisées, différentes selon les cadres sociodiscursifs et les finalités de l'action langagière. Chaque discipline scolaire a élaboré ses propres genres, par exemple le rapport d'expérimentation en sciences, la démonstration mathématique, la dissertation historique, le commentaire littéraire ou le résumé d'un récit. Ce sont tous des genres scolaires distincts quant à leur finalité, à leur contexte de production-réception, à leur forme linguistique et rhétorique, et au monde évoqué. Ces genres disciplinaires scolaires, fruits de transpositions conscientes ou non, sont des outils composites relevant à la fois des pratiques sociales, dont les pratiques discursives scientifiques, et des pratiques scolaires; ils constituent des «variantes propres à une communauté discursive spécifique, à une communauté d'instruction» (Bernié, 2002).

Différents didacticiens se sont intéressés aux caractéristiques génériques des genres disciplinaires scolaires (Aster, 2001; Astolfi, 1986; Astolfi, Peterfalvi \& Vérin, 1991; Audigier, 1998; Leroy, Gemenne \& Romainville, 1998; Simard, 2001; Vérin, 1998; O’Toole, 1996) et ont montré qu'ils possèdent des particularités linguistiques et textuelles (densité lexicale et syntaxique, etc.) qui constituent souvent un obstacle à leur appropriation par les élèves. Ce qu'on appelle la maitrise de la langue «se présente de manière particulière dans chaque champ conceptuel [et] déborde largement les objectifs de l'enseignement du français» (Astolfi, 1986, p. 56). C'est pourquoi le développement des capacités à comprendre et à produire les genres oraux et écrits disciplinaires ne peut pas relever uniquement de la discipline scolaire français. Cela, d'une part, parce que l'objet 
d'enseignement-apprentissage spécifique du français est la langue comme système ainsi que le développement de compétences discursives en lien avec les principales pratiques sociales du langage, dont celles rattachées à la littérature, et que, d'autre part, les genres disciplinaires sont produits par des «communautés discursives» particulières et ne peuvent, par conséquent, être efficacement étudiés que dans leur contexte de production-réception, c'est-à-dire dans chaque discipline. Aussi est-il nécessaire que les enseignants de chaque discipline soient mieux outillés pour développer chez leurs élèves les capacités langagières spécifiques à leur discipline. Mais afin de proposer des pistes d'intervention pertinentes aux enseignants, il est nécessaire de savoir et de comprendre ce qui se fait en classe, d'où le caractère essentiellement descriptif et analytique de notre démarche d'investigation qui, précisons-le, n'a aucune intention évaluative ou prescriptive.

\section{La démarche méthodologique}

L'enquête est menée sur une grande échelle à l'aide de trois outils d'investigation.

\section{Les outils d'investigation et l'échantillon}

Nous avons choisi les disciplines scolaires d'histoire et de sciences parce qu'au Québec elles s'enseignent à chaque cycle du secondaire ${ }^{6}$ (en 2 e secondaire pour le premier cycle et en $4 \mathrm{e}$ secondaire pour le 2e cycle) et que les activités de lecture et d'écriture qu'on y mène mettent en jeu des capacités langagières différentes puisque les genres de textes à lire et à écrire dans ces disciplines présentent des particularités génériques (discursives, textuelles, linguistiques et graphiques) distinctes.

Devant l'absence de données de recherches sur les pratiques de lecture et d'écriture dans les classes de sciences et d'histoire du secondaire au Québec, nous avons voulu recueillir des informations sur un bon nombre de dimensions de ces pratiques, ce qui nous a amenés à choisir le questionnaire (un destiné aux enseignants et un autre, aux élèves) comme premier et principal outil de collecte de données. Deux outils complémentaires sont utilisés successivement: des séquences vidéos d'enseignement de quatre à six périodes de cours d'histoire (quatre classes) et de sciences (quatre classes); des entretiens avec les enseignants concernés et un souséchantillon de leurs élèves, de milieux et de profils scolaires différents ${ }^{7}$.

L'enquête a été menée dans 50 écoles secondaires de la grande région de Québec $^{8}$. Des quelque 360 enseignants sollicités, 100 ont répondu à notre questionnaire. Plusieurs se sont dits disposés à nous "prêter» leur classe pour la passation du questionnaire à leurs élèves (nommé désormais Qél). L'échantillon compte 1150 élèves de classes mixtes de 2e et de 4 e secondaire ${ }^{9}$ (soit 617 filles et 533 garçons) de sept écoles de différents milieux et offrant des programmes d'études variés. Sans être statistiquement représentatif, cet échantillon fournit des données pertinentes sur la réalité scolaire du Québec. 


\section{L'élaboration et validation du Qél}

L'élaboration d'un questionnaire, sa validation puis sa mise en forme sont des tâches qui impliquent de nombreux choix méthodologiques. Nous présentons ceux relatifs au Qél, qui résultent, comme toujours, de compromis sur l'étendue et la profondeur du domaine étudié: thèmes retenus, nombre de questions et temps demandé aux enquêtés (Grawitz, 2001). Compte tenu des caractéristiques de nos sujets (des jeunes de 12 à 17 ans), de la nécessité de pouvoir analyser statistiquement nos données avec les ressources à notre disposition et, enfin, de la complémentarité des autres outils de l'enquête (questionnaire enseignants, séquences filmées et entrevues), nous avons opté pour un questionnaire combinant des questions fermées à choix multiple et des questions à échelle. L'expérience montre que les élèves sont plus enclins à répondre à un questionnaire demandant peu de temps et d'efforts. De plus, le traitement de données issues de questions fermées est plus simple, plus rapide et moins couteux que celui de données recueillies à l'aide de questions ouvertes (Grawitz, 1972/2001; Kornhauser \& Sheastley, 1977). Gauthier (1984) soutient que les questions fermées, «lorsqu'elles sont formulées avec soin, apparaissent tout aussi (et même parfois plus) valables que les questions ouvertes», sans compter que les deux autres instruments d'investigation utilisés permettront d'atténuer les inévitables faiblesses inhérentes aux questions fermées et d'enrichir les données.

Le questionnaire a été travaillé collectivement par les membres de l'équipe de recherche, qui ont mis à contribution leur expérience, leur culture langagière et leur point de vue (Grawitz, 2001) ${ }^{10}$. Ce travail a donné lieu à plusieurs versions du questionnaire, dont les dernières ont été révisées à l'aide d'une grille inspirée de celle de Kornhauser et Sheatsley (1977). Le questionnaire a été ensuite soumis à la critique de six spécialistes de différents milieux et niveaux d'expertise: des didacticiens des sciences, de l'histoire et du français, des enseignants de sciences et d'histoire du secondaire, des spécialistes de la langue et une dizaine d'adolescents. Puis le Qél a été soumis pour validation à six groupes d'élèves (165 élèves) de degrés différents provenant de deux écoles secondaires. La validation a permis d'effectuer des modifications à sa structure, à l'ordre des questions et à la langue utilisée.

Au cours de l'hiver et du printemps 2005, nous avons administré le Qél suivant un protocole. Deux membres de l'équipe en effectuaient ensemble la passation. L'enseignant quittait la salle après avoir introduit les chercheurs, qui procédaient à une brève présentation de la recherche en insistant sur l'importance de la contribution des élèves à l'avancement de la science, sur la confidentialité des réponses et sur le fait qu'il n'y avait pas de bonnes ou de mauvaises réponses (Grawitz 1972/2001; Cook, Selltiz \& Wrightsman, 1977). Les chercheurs aidaient la classe à remplir la fiche de renseignements personnels (âge, sexe, degré, programme) et faisaient la lecture des consignes. La passation durait entre 20 et 30 minutes. La plupart des élèves ont pris la tâche au sérieux et rares sont ceux qui semblent l'avoir bâclée. Les 1150 Qél ont été codés, saisis, puis traités avec le logiciel de statistiques SPSS. 
Trois variables sont prises en compte: le degré scolaire, la discipline et le sexe des élèves ${ }^{11}$. Toutes les questions à choix multiple ont été mises en relation avec chacune de ces variables de manière à identifier non seulement les grandes tendances, mais également les corrélations entre les variables.

\section{Les résultats du questionnaire élèves sur la fonction épistémique de l'écriture et les genres produits}

Les données obtenues par le traitement statistique du Qél nous fournissent des informations intéressantes sur les quatre dimensions du rapport à l'écrit (RÉ) des élèves comme l'indique le tableau 1, qui synthétise les questions du questionnaire pour chacune de ces dimensions.

\section{Tableau 1: Présentation synthétique du Qél}

\begin{tabular}{|l|l|c|}
\hline Dimension du RÉ & Thème des questions & $\begin{array}{c}\text { Nombre de } \\
\text { questions }\end{array}$ \\
\hline Affective & $\begin{array}{l}\text { Fréquence des pratiques et temps accordé aux pratiques de L/É en } \\
\text { dehors de l'école }\end{array}$ & 4 \\
\hline & Cours où la L/É est jugée plus intéressante & 2 \\
\hline & Attitude par rapport à sa compréhension en lecture & 2 \\
\hline & Attitude par rapport à la qualité du texte à écrire & 2 \\
\hline & Jugement sur ses propres difficultés en L/É & 2 \\
\hline Axiologique & Préférences quant aux genres de lecture en classe & 3 \\
\hline & Maitrise socialement souhaitable de la L/É & 2 \\
\hline & Jugement sur l'importance que les enseignants accordent à la L/É & 1 \\
\hline Praxéologique & Importance de corriger son texte & 2 \\
\hline & Importance de bien comprendre un texte & 5 \\
\hline Cognitive & Les usages de la L/É en classe & 2 \\
\hline & Importance de la L/É pour apprendre & 3 \\
\hline & Genres de textes lus ou écrits & 2 \\
\hline
\end{tabular}

Légende: L (lecture), É (écriture)

Vu l'espace limité, nous consacrerons la suite de cet article aux résultats obtenus à deux questions qui sont au cœur de notre problématique: la première concerne les perceptions que les élèves se font du rôle ou de la fonction de leurs pratiques 
d'écriture en histoire et en sciences: elle jette un éclairage sur la conception que les élèves se font de la fonction épistémique de l'écriture; la deuxième nous informe sur les genres de textes produits par ces élèves, livrant des informations sur les genres impliqués dans ces disciplines ${ }^{12}$.

\section{La fonction épistémique de l'écriture}

Les élèves de notre échantillon se sont vu demander, à la question 18 , à quoi leur sert d'écrire en histoire et en sciences. La question comporte une liste de huit énoncés placés de façon aléatoire, couvrant les quatre fonctions de l'écriture que nous avons catégorisées comme suit.

Tableau 2: Répartition des énoncés de la question 18 selon la fonction dominante de l'écriture

\begin{tabular}{|l|l|}
\hline Fonction de l'écriture & $\begin{array}{l}\text { Énoncés proposés } \\
\text { À quoi te sert d'écrire en sciences, en histoire? }\end{array}$ \\
\hline Communicative utilitaire (FCU) & à répondre à des questions d'examen, de devoir \\
\hline Mémorielle utilitaire (FMU) & $\begin{array}{l}\text { à noter ce que tu dois retenir } \\
\text { à te souvenir de ce que tu dois faire }\end{array}$ \\
\hline Épistémique (FÉ) & $\begin{array}{l}\text { à mieux comprendre ce que tu dois apprendre; } \\
\text { à taider à retenir ce que tu écris } \\
\text { à préparer des travaux (recherches, exposés, etc.) } \\
\text { à préciser tes idées, réfléchir }\end{array}$ \\
\hline Créatrice (FC) & $\grave{a}$ développer ton imagination \\
\hline
\end{tabular}

La fonction communicative vise des fins strictement utilitaires (FCU): l'élève se sert de l'écrit pour montrer ce qu'il sait, le plus souvent dans un but d'évaluation (examens, tests), il s'agit de restituer des informations ou des connaissances par écrit et non d'en élaborer. La fonction mémorielle utilitaire (FMU): l'élève écrit ce qu'il doit faire dans son agenda pour s'en souvenir (date des examens, remise des travaux) et note dans son cahier d'exercices ou sur des feuilles remises par l'enseignant, le plus souvent sous la dictée, des informations. En fait, il doit compléter des énoncés et compléter des informations de ce qu'il faut retenir; il n'y a pas de production discursive personnelle à proprement parler. La fonction épistémique (FÉ) comprend quatre items: l'élève considère que l'écriture lui permet de mieux comprendre ce qu'il doit apprendre, l'aide à retenir ce qu'il doit savoir, car il comprend que, de les avoir écrites, les informations s'imprègnent mieux, se stockent dans sa mémoire et pourront plus facilement être sollicitées; l'écriture lui sert aussi à préparer des travaux (prise de notes des éléments importants des documents consultés, par exemple) et, enfin, l'écriture joue un rôle dans la précision des idées et lui permet de réfléchir. Enfin, la fonction créatrice (FC) renvoie au fait que l'écriture permet de développer son imagination. Cependant, ce regroupement ne doit pas être interprété de façon stricte. Dans une même acti- 
vité d'écriture, une fonction peut coexister avec une autre fonction. Ainsi, si l'activité «préparer des travaux (recherches, exposés, etc.)» met en jeu les fonctions épistémique et créatrice de l'écriture, elle remplit aussi un rôle de communication utilitaire puisque, au bout du compte, l'élève devra communiquer - soit à l'oral, soit à l'écrit - le fruit de son travail à l'enseignant en vue d'une évaluation. Il reste que le processus d'élaboration de ce travail (chercher de l'information, la sélectionner, l'organiser et, éventuellement, rédiger un texte) consiste en une série d'opérations cognitives de haut niveau qui mettent en ouvre la fonction épistémique de l'écrit. Les élèves, à chacun des énoncés, devaient répondre sur une échelle à quatre degrés (jamais, parfois, assez souvent, très souvent).

Le tableau 3 présente les résultats obtenus à la question 18 selon les variables sexe et degré scolaire. Les résultats sont donnés dans l'ordre décroissant - légèrement différent en histoire et en sciences - de la somme des fréquences assez souvent et très souvent ${ }^{13}$. Le $\mathrm{V}$ de Cramer mesure l'intensité de la relation pour la variable sexe; le coefficient de Spearman ${ }^{14}$ mesure l'intensité de la relation pour la variable degré scolaire. Les astérisques indiquent le niveau de signification de la relation $(\mathrm{p})$.

La colonne «Ensemble des cas» présente les résultats des réponses données par l'ensemble des élèves en pourcentage. Deux usages de l'écriture se distinguent nettement: selon les élèves, l'écriture sert le plus souvent, pour $90 \%$ d'entre eux, à répondre à des questions d'examen, de devoir (FCU), puis, pour $85 \%$, à noter ce qui doit être retenu (FMU), deux des fonctions essentiellement utilitaires, faisant peu ou pas intervenir la FÉ compte tenu de la façon dont ces pratiques sont menées, comme nous le verrons plus loin.

À l'inverse, deux autres perceptions des usages de l'écriture se distinguent par leur faible fréquence: écrire pour préciser des idées, réfléchir (FÉ) et écrire pour développer son imagination (FC). Moins de la moitié des élèves (39\% des élèves en histoire et $48 \%$ en sciences) considère qu'écrire leur permet de préciser leur pensée, de réfléchir, énoncé qui nous semble le mieux illustrer la fonction épistémique de l'écrit, car l'appropriation des savoirs passe par une attitude réflexive. Et seulement le quart affirme que l'écriture dans ces disciplines leur permet de développer leur imagination (FC). On observe toutefois une relation statistiquement significative de forte intensité entre ces variables (information absente du tableau). Ainsi, la plupart des élèves qui affirment que l'écriture permet de préciser ses idées et de réfléchir disent également qu'elle est un moyen de développer son imagination ${ }^{15}$. La reconnaissance de ces deux fonctions par les mêmes élèves montre qu'entre le quart et la moitié des élèves ont une conception de l'écrit qui dépasse une conception utilitaire, pragmatique.

En effet, si on considère les pourcentages de réponses aux énoncé trois, quatre et cinq du tableau 3, on constate qu'entre $68 \%$ et $73 \%$ des élèves en histoire et en sciences perçoivent la fonction épistémique de l'écriture, ce qui n'est pas négligeable, bien qu'on ne peut guère préciser la teneur de cette perception. 
Tableau 3: Fonctions de l'écriture en histoire et en sciences pour des élèves du secondaire selon le sexe et le de degré scolaire

\begin{tabular}{|c|c|c|c|c|c|c|c|}
\hline \multirow{3}{*}{$\begin{array}{l}\text { À quoi sert d'écrire en histoire et en } \\
\text { sciences? Résultats assez souvent et } \\
\text { très souvent }\end{array}$} & \multirow{3}{*}{$\begin{array}{c}\text { En- } \\
\text { semble } \\
\text { des cas } \\
(\%)\end{array}$} & \multicolumn{2}{|c|}{ Sexe } & \multicolumn{4}{|c|}{ Degré } \\
\hline & & \multicolumn{2}{|c|}{$(\%)$} & \multicolumn{3}{|c|}{$(\%)$} & Spearman \\
\hline & & $\mathrm{F}$ & G & & $2 \mathrm{e}$ & $4 \mathrm{e}$ & \\
\hline \multicolumn{8}{|l|}{ En histoire, écrire sert à... } \\
\hline Répondre (examen, devoir) - FCU & 90 & 93 & 87 & $0,128^{* * *}$ & 90 & 90 & $-0,058^{* *}$ \\
\hline Noter pour retenir - FMU & 85 & 91 & 80 & $0,234^{* * *}$ & 80 & 91 & $0,233^{* * *}$ \\
\hline Mieux comprendre - FÉ & 73 & 79 & 66 & $0,192^{* * *}$ & 66 & 77 & $0,132^{* * *}$ \\
\hline Aider à retenir - FÉ & 72 & 77 & 66 & $0,165^{* * *}$ & 66 & 75 & $0,134^{* * *}$ \\
\hline Préparer des travaux - FÉ & 68 & 70 & 67 & $0,087^{* *}$ & 80 & 60 & $-0,232^{* * *}$ \\
\hline Se souvenir - FMU & 67 & 72 & 62 & $0,154^{* * *}$ & 69 & 66 & 0,027 \\
\hline Préciser les idées, réfléchir - FÉ & 39 & 42 & 35 & $0,078^{*}$ & 43 & 37 & $-0,034$ \\
\hline Développer l'imagination - FC & 24 & 23 & 29 & $0,128^{* * *}$ & 29 & 24 & $-0,030$ \\
\hline \multicolumn{8}{|l|}{ En sciences, écrire sert à... } \\
\hline Répondre (examen, devoir) - FCU & 90 & 92 & 87 & $0,140^{* * *}$ & 89 & 90 & $-0,048$ \\
\hline Noter pour retenir - FMU & 85 & 89 & 81 & $0,216^{* * *}$ & 79 & 89 & $0,158^{* * *}$ \\
\hline Mieux comprendre - FÉ & 73 & 79 & 67 & $0,185^{* * *}$ & 69 & 77 & $0,124^{* * *}$ \\
\hline Préparer des travaux - FÉ & 70 & 73 & 66 & $0,119^{* * *}$ & 75 & 66 & $-0,117^{* * *}$ \\
\hline Aider à retenir $-\mathrm{FÉ}$ & 68 & 73 & 62 & $0,188^{* * *}$ & 65 & 70 & $0,088^{* * *}$ \\
\hline Se souvenir - FMU & 66 & 70 & 61 & $0,135^{* * *}$ & 66 & 65 & 0,021 \\
\hline Préciser les idées, réfléchir - FÉ & 48 & 52 & 43 & $0,100^{* * *}$ & 52 & 45 & $-0,041$ \\
\hline Développer l'imagination - FC & 24 & 23 & 26 & 0,072 & 26 & 23 & $-0,015$ \\
\hline$(\mathrm{N})$ & $(1150)$ & (617) & (533) & & (464) & (686) & \\
\hline
\end{tabular}

\section{Différences entre les sexes}

L'ordre des priorités dans les usages de l'écriture est le même pour les filles et les garçons. On note toutefois de nombreuses différences - faibles et modérées entre les sexes: pour chaque énoncé, à l'exception d'écrire pour développer l'imagination, les filles sont plus nombreuses à répondre par assez souvent et très sou- 
vent. Pour ce résultat, on peut toutefois se demander si les sujets ont vraiment limité leur réponse à l'écriture en histoire et en sciences ou s'ils n'ont pas plutôt pensé à l'écriture en général, à l'école, incluant le cours de français, car on ne trouve pas beaucoup de traces de genres produits qui feraient appel à l'imagination en sciences et en histoire (voir plus bas). Les filles disent écrire plus souvent que les garçons dans le but de répondre à des questions d'examen et de devoir. Considérant que les élèves ont certainement à faire les mêmes examens, la différence doit représenter plutôt le temps consacré à faire les devoirs: les filles les feraient plus souvent. Cette hypothèse viendrait d'ailleurs appuyer les travaux qui montrent qu'à l'école la plupart des filles sont plus «scolaires», plus appliquées et disciplinées que les garçons, ce qui expliquent, entre autres, leur plus grande réussite scolaire. En effet, elles sont plus nombreuses à noter ce qu'il faut faire et retenir que les garçons.

De plus, pour tous les items relevant de la fonction épistémique, les filles se démarquent encore des garçons. Il y a un écart de plus ou moins $10 \%$ entre les sexes. Ces données ajoutées à celles concernant le rapport affectif à l'écrit (Chartrand, Prince \& Blaser, à paraître) et à la plus grande valeur que les filles accordent à l'écrit "pour réussir et s'épanouir dans la société» (question 8 du Qél) montrent que leur rapport à l'écrit est, statistiquement, plus impliqué ou vécu plus positivement que celui des garçons: elles ont plus de pratiques, ces dernières sont plus diversifiées, elles y consacrent plus de temps volontairement; elles ont plus de gout pour la lecture et l'écriture, valorisent davantage ces pratiques et sont davantage conscientes des diverses fonctions de l'écriture.

\section{Différences entre les degrés scolaires}

Quant aux différences entre les degrés scolaires, en histoire les élèves de $4 \mathrm{e}$ secondaire prendraient plus souvent des notes que leurs cadets (91\% contre 80 $\%)$, mais ils écriraient moins pour rédiger des travaux (60\% contre $80 \%)$. Les contraintes pesant sur le cours d'histoire de 4 e secondaire y sont sans doute pour beaucoup. L'examen ministériel qui couronne ce cours, et dont la réussite est nécessaire à l'obtention du diplôme d'études secondaires, est un examen portant essentiellement sur des contenus factuels. Il contient un très grand nombre de questions, majoritairement à choix multiple, sur un contenu très vaste (l'histoire du Québec et du Canada). La préparation des élèves à cette épreuve semble passer par un enseignement magistral de type notionnel et évènementiel où les élèves doivent prendre beaucoup de notes (le plus souvent sous la dictée), mais qui laisse peu de temps pour la production de travaux personnels de recherches, d'exposés, autrement dit à des activités où la fonction épistémique de l'écriture est mise à contribution. Au contraire, le cours d'histoire de 2e secondaire, qui n'est pas sanctionné par un examen, laisse plus de latitude aux enseignants qui en profitent souvent pour faire mener par leurs élèves des recherches et des projets qui demandent des activités de lecture et d'écriture plus élaborées. Il y a $20 \%$ de plus d'élèves de $2 \mathrm{e}$ qui disent écrire pour préparer des travaux. Par contre, fait 
rassurant peut-être, il semble que les élèves de $4 \mathrm{e}$ secondaire soient un peu plus conscients de la fonction épistémique de l'écrit (écart de $10 \%$ ) que les plus jeunes: le passage par l'écriture permettrait, selon eux, d'améliorer sa compréhension et de faciliter la rétention d'information. Est-ce dû à l'âge, au cheminement scolaire, au fait qu'ils ont des pratiques langagières plus variées, à ces facteurs réunis ? Difficile de répondre à partir de ces données d'enquêtes par questionnaire, mais c'est une question que nous explorerons lors des entretiens avec des élèves, dans une phase ultérieure de la recherche.

Les mêmes tendances s'observent en sciences. Les élèves de $4 \mathrm{e}$ secondaire disent prendre aussi plus de notes que les élèves de $2 \mathrm{e}$ secondaire et faire moins de travaux, mais les écarts entre les premiers et les seconds sont moindres qu'en histoire; alors qu'ils étaient de $20 \%$ en histoire, ils ne sont que de $9 \%$ en sciences. Cela s'explique sans doute par la place qu'y prennent les expériences de laboratoire qui donnent lieu parfois à des travaux écrits un peu plus élaborés. Mais ici encore, l'examen obligatoire de sciences en $4 \mathrm{e}$ secondaire semble peser sur la latitude des enseignants à proposer diverses pratiques d'écriture.

\section{Les genres de textes produits}

Afin d'en savoir plus sur les genres de textes produits en histoire et en sciences, nous avons demandé aux élèves quels genres d'écrits ils rédigeaient dans ces disciplines et à quelle fréquence (jamais, parfois, assez souvent et très souvent). Pour le cours d'histoire, trois genres étaient suggérés, suite à la préenquête qui indiquait que c'étaient les genres les plus pratiqués et les seuls genres existants dans ces cours: le résumé, la recherche, les réponses à des exercices ou à des questions d'examen; pour le cours de sciences s'ajoutait le rapport d'expérience. Le tableau 4 présente les résultats fusionnés des catégories assez souvent et très souvent ${ }^{16}$.

L'examen du tableau 4 confirme qu'en histoire les élèves auraient pour principale activité d'écriture la rédaction de réponses à des questions $(83 \%)$, que ce soit dans le cadre d'un exercice ou d'un examen, ce qui est congruent avec le rôle qu'ils jugent le plus courant de leurs activités d'écriture (question 18). Suit loin derrière la production de résumés $(39 \%)$ et de recherches $(27 \%)$. On note également que les filles disent répondre plus souvent à des questions que les garçons (comme pour la question 18). Les deux autres genres (le résumé et la recherche) sont rédigés régulièrement par moins de la moitié des élèves, dans des proportions quasi égales chez les garçons et les filles.

Concernant les degrés scolaires, on observe une différence importante dans la fréquence de rédaction des recherches selon que les élèves sont en $2 \mathrm{e}$ ou en $4 \mathrm{e}$ secondaire. Les élèves de $4 \mathrm{e}$ écriraient beaucoup moins pour préparer des travaux que ceux de $2 \mathrm{e}$, comme ils rédigeraient beaucoup moins de recherches que leurs cadets $(13 \% \text { contre } 48 \%)^{17}$. On constate que les réponses convergent avec celles de la question 18 et que l'explication est sans doute en partie la même (exigences curriculaires plus importantes en 4e), mais il faudrait aussi savoir si les élèves de $2 \mathrm{e}$ et de $4 \mathrm{e}$ secondaire ont la même représentation de ce qu'est faire une 
recherche, si ce terme est jugé différent de produire une dissertation historique, par exemple.

Tableau 4: Types d'écrits rédigés assez souvent et très souvent en sciences et en histoire depuis le début de l'année scolaire

\begin{tabular}{|c|c|c|c|c|c|c|c|}
\hline & & & & & $\begin{array}{r}\text { Degré s } \\
(\%\end{array}$ & blaire & \\
\hline & $\begin{array}{c}\text { Ensemble } \\
\text { des cas } \\
(\%)\end{array}$ & $\mathrm{F}$ & G & $\begin{array}{l}\text { V de } \\
\text { Cramer }\end{array}$ & $2 \mathrm{e}$ & $4 \mathrm{e}$ & $\begin{array}{c}\text { Coeffi- } \\
\text { cient de } \\
\text { Spearman }\end{array}$ \\
\hline $\begin{array}{l}\text { Type d'écrit rédigé en } \\
\text { Réponses à des questio }\end{array}$ & & & & & & & \\
\hline (exercice, examen) & 83 & 88 & 78 & $0,132^{* * *}$ & 84 & 83 & $-0,048$ \\
\hline Résumés & 39 & 42 & 36 & $0,075^{*}$ & 41 & 38 & $-0,037$ \\
\hline Recherches & 27 & 28 & 26 & 0,069 & 48 & 13 & $-0,404^{* * *}$ \\
\hline $\begin{array}{l}\text { Type d'écrit rédigé en } \\
\text { Réponses à des questio }\end{array}$ & & & & & & & \\
\hline (exercice, examen) & 80 & 85 & 75 & $0,172^{* * *}$ & 80 & 81 & 0,001 \\
\hline Rapport d'expérience & 77 & 83 & 70 & $0,156^{* * *}$ & 85 & 71 & $-0,186^{* * *}$ \\
\hline Résumés & 26 & 28 & 24 & 0,055 & 26 & 26 & 0,014 \\
\hline Recherches & 25 & 25 & 25 & $0,119^{* *}$ & 30 & 22 & $-0,069^{* * *}$ \\
\hline$(\mathrm{N})$ & (1150) & (617) & (533) & & (464) & (686) & \\
\hline
\end{tabular}

En sciences, le genre Réponses à des questions domine également, mais il est suivi de très près (3 points d'écart) par un genre disciplinaire canonique, le rapport d'expérience ou de laboratoire. On note là aussi une différence entre les garçons et les filles, qui déclarent s'adonner plus fréquemment à produire ce genre que les garçons $(85 \%$ contre $71 \%)$. Les rapports d'expérience étant souvent à faire ou à finir en devoir, on peut avancer que la différence entre les sexes s'explique par le fait que les garçons ont moins tendance à faire leurs devoirs que les filles. Il y a aussi une différence de faible intensité entre les degrés pour ce qui est de la rédaction des rapports d'expérience: ce genre est moins fréquemment rédigé en $4 \mathrm{e}$ qu'en 2e secondaire. Sachant que les élèves de 4e secondaire subissent un examen de laboratoire en fin d'année, on aurait pu s'attendre à ce qu'ils fassent davantage, ou au moins autant, de rapports d'expérience en cours d'année que les 
élèves de 2e secondaire. Ils sont tout de même $71 \%$ à dire en faire assez souvent et très souvent, ce qui confirme que le genre rapport de laboratoire est un genre dominant en sciences au secondaire. Nos vidéos de séquences de cours révèlent que la rédaction du rapport de laboratoire ne constitue pas un travail discursif important pour les élèves. En fait, ces derniers doivent le plus souvent remplir un protocole déjà fortement balisé, qui laisse peu de place à la production personnelle d'écrits et à la réflexion. De façon générale, l'écriture sert à consigner des informations pour les mémoriser beaucoup plus qu’à structurer la pensée ou à générer des idées nouvelles et à construire des savoirs. Nous pouvons réaffirmer que la fonction épistémique de l'écrit ne semble pas constituer un enjeu important dans les classes d'histoire et de sciences du secondaire.

Mis à part le rapport d'expérience en sciences, peut-on parler de genres textuels propres à chaque discipline dans le cas de réponses à des questions d'exercices ou d'examens, de résumés et de recherches ? Il semble plutôt qu'il s'agisse de genres scolaires passe-partout, que chaque enseignant façonne à son gré, par des consignes plus ou moins précises. D'après nos observations en histoire, la production d'une recherche en 2e secondaire correspond plutôt à un texte de type descriptif sur un thème donné, alors que celle de $4 \mathrm{e}$ relève plutôt d'un texte de type explicatif sur un phénomène ou évènement. Quant aux résumés, ils varient énormément de quelques lignes à quelques pages. Les seuls exemples que nous ayons de recherches en sciences porte sur un portrait de scientifique. La suite de la recherche, qui comprendra aussi des analyses de productions d'élèves dans les classes où des séquences de cours ont été filmées (classes exemplaires !) nous renseignera sur les spécificités génériques des textes produits.

\section{Conclusion}

Ces premiers résultats confortent, en les objectivant, des impressions ou des intuitions répandues chez les enseignants et les chercheurs en éducation. Les pratiques de lecture et d'écriture des élèves de 12 à 17 ans sont peu variées et peu fréquentes, sauf celles réalisées à l'aide de supports informatiques, qui semblent principalement répondre à des besoins communicationnels, voire phatiques entre adolescents. Par ailleurs, bien que la lecture et l'écriture occupent une partie non négligeable du temps dans les cours d'histoire et de sciences, ces activités ne semblent pas prisées des élèves, qui perçoivent peu leur fonction épistémique et leur impact sur leur réussite dans ces disciplines. Ils disent lire et écrire à des fins strictement utilitaires et à court terme: noter ce qu'il faut faire, apprendre et retenir en fonction des examens. Les écrits réalisés (répondre à des questions, prendre des notes, faire des résumés, etc.) ne semblent pas présenter de spécificités disciplinaires, si ce n'est le rapport de laboratoire, ni être perçus comme très exigeants au plan discursif. En outre, on note un léger écart entre les filles et les garçons, de même qu'entre les élèves inscrits dans des programmes plus exigeants et plus sélectifs et ceux des programmes réguliers, et on remarque que l'importance de l'écrit décroit de la 2e à la 4e secondaire. Ces résultats assez inquiétants, 
vont dans le sens d'autres données d'enquête (Lebrun, 2004 et l'enquête quinquennale du ministère de la Culture et des Communications du Québec commentée par Boileau, 2005). De plus, ils sont congruents avec les résultats obtenus par le questionnaire enseignants de notre recherche. Les entretiens semi-dirigés et les analyses des écrits scolaires d'élèves, que nous réaliserons dans une étape ultérieure de l'étude, nous permettront de mieux interpréter ces résultats.

Bien que la recherche dont nous venons d'exposer une petite partie des résultats se situe dans le domaine de la didactique du français, nous considérons qu'elle fournit des données intéressantes aux chercheurs d'autres domaines des sciences de l'éducation, en premier lieu celui des didactiques d'histoire et de sciences, en indiquant comment enseignants et élèves pensent et vivent le rapport à l'écrit, en particulier pour développer les connaissances et habiletés propres à ces univers disciplinaires. Plus globalement, elle devrait interpeller les chercheurs qui tentent de cerner la place du langage écrit dans les dispositifs d'enseignement-apprentissage, considérant que l'entreprise éducative est une activité fondamentalement culturelle dont la langue constitue à la fois l'assise et le mode d'accès privilégié.

\section{Notes}

1 Ce texte adopte l'orthographe rectifiée.

2 La recherche est menée par l'équipe de recherche Scriptura réunissant des étudiants des trois cycles universitaires sous la direction de la didacticienne du français Suzanne-G. Chartrand, professeure de la Faculté des sciences de l'éducation de l'Université Laval. La recherche fait l'objet d'un financement du Fonds québécois pour la recherche sur la société et la culture (FQRSC) et du Conseil de recherche en sciences humaines du Canada (CRSH) de 2004 à 2007; elle bénéficie en outre de la généreuse collaboration de J. Dolz et B. Schneuwly, professeurs à l'Université de Genève et responsables du GRAFE (Groupe de recherche et d'analyse du français écrit) financé par le Fonds national suisse.

3 Nous parlons de coconstruction, car, d'une part, la construction de la pensée, de la connaissance et des capacités langagières ne peut se faire que dans l'interaction sociale ( $\mathrm{Vy}$ gotski) et, d'autre part, ce concept met l'accent sur la valeur épistémologique de la construction des savoirs.

4 La fonction cognitive de l'écriture englobe un très grand nombre d'activités mentales susceptibles d'être mobilisées par l'écrit, dont la mémorisation, l'attention volontaire, l'anticipation, la conceptualisation, la schématisation. Dans cette recherche, nous nous intéressons uniquement aux activités cognitives en jeu dans l'écrit qui ont un lien direct avec la construction de savoirs disciplinaires, ce que nous nommons la fonction épistémique de l'écrit. Cette dernière fait intervenir un nombre plus circonscrit d'opérations mentales que la fonction cognitive.

5 Pour une lecture critique de ces mouvements, voir Ochsner \& Fowler, 2004.

6 Au moment de l'enquête, ces disciplines ne s'enseignaient qu'en 2e secondaire (8e année de scolarité) et en 4e secondaire (10e année).

7 Les vidéoscopies et les entretiens n'étant pas terminés, l'analyse ne tient compte que des questionnaires.

8 Notons que cette région est assez représentative du Québec par la variété des milieux (urbain, rural, semi-rural; favorisé et défavorisé; en majorité francophone) et qu'on y trouve des écoles publiques et privées offrant différents types de programmes d'études. 
$95 \%$ des répondants étaient des élèves de 5 e secondaire qui, pour diverses raisons, suivaient des cours d'histoire ou de sciences de $4 \mathrm{e}$ secondaire.

10 Avant de rédiger notre questionnaire, nous avons analysé des questionnaires de recherche portant sur des objets similaires (Barré-de Miniac \& Reuter 2000; Groupe DIEPE, 1995, Lafont-Terranova \& Colin 2002.

11 Nous ne disposons pas de données sur le milieu socioéconomique et culturel des élèves, cette variable ne faisant pas partie de l'enquête. Nous ne jugions pas réalistes de demander aux élèves de cet âge de nous fournir des données valables sur cette question complexe.

12 Bien qu'il s'agisse de pratiques déclarées, la cohérence dans les réponses des élèves ainsi que les données recueillies par le questionnaire enseignants permettent de penser que ces données sont près de la réalité.

13 Les tests et mesures de corrélation ont été effectués sur les données brutes, avant leur regroupement.

14 Le V de Cramer est une mesure basée sur le chi-carré, sa valeur se situe entre 0 et 1 ; le coefficient de Spearman est une mesure d'association pour variables ordinales ou d'intervalle, sa valeur se situe entre -1 et +1 . Dans notre analyse, nous considérons qu'une valeur absolue du V de Cramer ou du coefficient de Spearman comprise entre 0,100 et 0,199 indique une relation faible entre les variables; entre 0,200 et 0,399 , une relation modérée; et à partir de 0,400 , une relation forte.

15 En histoire, au niveau de signification 0,000, le coefficient de Spearman est de 0,494. En sciences, au même niveau de signification, le coefficient de Spearman est de 0,486.

16 Les tests et mesures de corrélation ont été effectués sur les données brutes, avant leur regroupement.

17 Un résultat différent aurait remis en question la cohérence des données, car la rédaction d'une recherche va forcément de pair avec la préparation de travaux.

\section{Références bibliographiques}

Ackerman, P.T. (1993). The promise of writing to learn. Written Communication, 10 (3), 334370 .

Aster (2001). Écrire pour comprendre les sciences. (33). Paris: Institut national de recherche pédagogique.

Astolfi, J.-P. (1986). Les langages et l'élaboration de la pensée scientifique. Le français aujourd'hui, 74, 51-57.

Astolfi, J.-P., Peterfalvi, B. \& Vérin, A. (1991). Compétences méthodologiques en sciences expérimentales. Paris: INRP.

Audigier, F. (1998). La description dans l'enseignement de l'histoire et de la géographie au plus près du réel. In Y. Reuter (Éd.), La description: théories, recherches, formation, enseignement (pp. 229-246). Lille: Presses Universitaires du Septentrion.

Bakhtine, M. (1984). Esthétique de la création verbale. Paris: Gallimard.

Barré-De Miniac, C. (1995). La didactique de l'écriture: nouveaux éclairages pluridisciplinaires et état de la recherche (Note de synthèse). Revue française de pédagogie, 113, 93-133.

Barré-De Miniac, C. (2002). Le rapport à l'écriture. Une notion à plusieurs dimensions. Pratiques, 113-114, 29-40.

Barré-De Miniac, C. \& Reuter, Y. (2000). Apprendre à écrire dans les différentes disciplines au collège (Présentation d'une recherche en cours). La lettre de la DFLM, 26, 18-23.

Bernié, J.-P. (2002). L'approche des pratiques langagières scolaires à travers la notion de communauté discursive: un apport à la didactique comparée ? Revue française de pédagogie 141, 77-88.

Boileau, J. (2005). Lire? A quoi bon!, éditorial du quotidien Le Devoir, p. A8, 18 novembre.

Bronckart, J.-P. (1996). Activité langagière, textes et discours: pour un interactionnisme socio-discursif. Lausanne: Delachaux et Niestlé. 
Bronckart, J.-P., Bain, D., Schneuwly, B., Davaud, C. \& Pasquier, A. (1985). Le fonctionnement des discours. Un modèle psychologique et une méthode d'analyse. Neuchâtel, Paris: Delachaux et Niestlé.

Chabanne, J.-C. \& Bucheton, D. (2002). Parler et écrire pour penser, apprendre et se construire. L'écrit et l'oral réflexifs. Paris: PUF.

Chartrand, S.-G. (2000). Le programme de français de 1995. Un outil pour développer la compétence d'écriture au secondaire. Québec français ( $\mathrm{n}^{\circ}$ hors série: La grammaire au coeur du texte), 24-27.

Chartrand, S.-G. \& Prince, M. (soumis). La dimension affective du rapport à l'écrit des élèves du secondaire québécois. Revue française de pédagogie.

Commander, N. \& Smith, B.D. (1996). Learning logs: a tool for cognitive monitoring. Journal of Adolescent and Adult Literacy, 39, 446-453.

Cook, S.W. Selltiz, C. \& Wrightsman, L.S. (1977). Les méthodes de recherche en sciences sociales. Montréal: Éditions HRW.

Delcambre, I., Dolz, J. \& Simard, S. (2000). Écrire pour apprendre: une activité complexe aux sens multiples. La lettre de la DFLM, 26, 3-5.

Gauthier, B. (Éd.). (1984). Recherche sociale. De la problématique à la collecte des données. Sillery: Les Presses de l'Université du Québec.

Goody, J. (1979). La raison graphique. La domestication de la pensée sauvage. Paris: Éditions de Minuit.

Grawitz, M. (1972/2001). Méthodes des sciences sociales. Paris: Dalloz.

Groupe DIEPE. (1995). Savoir écrire au secondaire. Étude comparative auprès de quatre populations francophones d'Europe et d'Amérique. Bruxelles: De Boek Université.

Halliday, M.A.K. \& Martin, J.R. (1993). Writing science: literacy and discursive power. London: Falmer.

Jaubert, M., Rebière, M. \& Bernié, B. (2003). Construction des connaissances et langage dans les disciplines d'enseignement. Actes du colloque pluridisciplinaire international. Bordeaux (3-5 avril 2003) [CD-Rom].

Kornhauser, A. \& Sheatsley, P.B. (1977). L'élaboration des questionnaires et les techniques d'interview. In S. W. Cook, C. Selltiz \& L. S. Wrightsman (Éd.), Les méthodes de recherche en sciences sociales. Montréal: Éditions HRW.

Lafont-Terranova, J. \& Colin, D. (2002). Les enseignants de collège et l'écriture: des déclarations aux représentations. Pratiques, 115-116, 167-180.

Langer, J. \& Applebee, A. (1987). How writing shapes thinking. Urbana Illinois: NCTE.

Lebrun, M. (sous la dir.)(2004). Les pratiques de lecture des adolescents québécois. Sainte Foy: Éd. Multimondes

Leroy, A., Gemenne, L. \& Romainville, M. (Éd.) (1998). Écrire pour apprendre: activités d'éveil et de sciences. Cahiers du SEGEC, (9).

Mc Crindle, A.R. \& Christensen, C.A. (1995). The impact of learning journals on metacognitive and cognitive processes and learning performance. Learning and Instruction, 5, $167-185$.

O’Toole, M. (1996). Science, schools, children and books: exploring the classroom interface between science and language. Studies in Science Education, 28, 113-143.

Ochsner, R. \& Fowler, J. (2004). Playing Devil's Advocate: Evaluating the literature of the WAC/WID movement. Review of Educational Research, 74 (2), 117-140.

Peytard, J. (1970). Oral et scriptural: deux ordres de situations et de descriptions linguistiques. Langue française, 6, 35-47.

Reuter, Y. (2001). Vers une didactique de l'écriture: retour sur quelques propositions. Conférence prononcée à Montréal dans le cadre d'une journée étude de la DFLM, section Québec/Canada. Manuscrit inédit.

Rowell, P.M. (1997). Learning in school science: The promise and practices of writing. Studies in Science Education, 30, 19-56. 
Schneuwly, B. (1988). Le langage écrit chez l'enfant: la production de textes informatifs et argumentatifs. Neuchâtel: Delachaux et Niestlé.

Schneuwly, B. (1995). Apprendre à écrire. Une approche socio historique. In J.-Y. Boyer, J.-P. Dionne \& P. Raymond (Éd.), La production de textes (pp. 73-100). Montréal: Les Éditions Logiques.

Simard, C. (2000). Aperçu des études anglo-saxonnes sur le rôle de l'écriture dans l'apprentissage. La Lettre de la DFLM, 26, 18-23.

Simard, C. (2001). Les compétences langagières dans les disciplines scolaires. Québec français, $123,18-23$.

Sutton, C.R. (1995). Quelques notions sur l'écriture et la science: une vue personnelle d'outre-Manche. Repères, 12, 37-52.

Vérin, A. (1998). La description dans l'enseignement des sciences expérimentales. In Y. Reuter (Éd.), La description: théories, recherches, formation, enseignement (pp. 247-262). Paris: Presses Universitaires du Septentrion.

Vygotski, L. (1997/1934). Pensée et langage. (F. Sève, trad.) Paris: La Dispute.

Mots clés: préparation à l'écriture, programme de cours, évaluation, enseignement secondaire

\section{Die epistemische Funktion des Schriftlichen im Geschichts- und Naturwissenschaftsunterricht. Eine Untersuchung an Klassen der Sekundarstufe I in Québec}

\section{Zusammenfassung}

Die Reformen der schulischen Curricula berücksichtigen die zentrale Funktion von Sprache im Aufbau und der Ausformung des Wissens bei Schülerinnen und Schülern. Welche Rolle übernimmt dabei das Schriftliche $\neg$ schriftliche Unterlagen wie auch zu erstellende Texte $\neg$ etwa im Geschichtsunterricht und im naturwissenschaftlichen Unterricht an Klassen der Sekundarstufe I? In einer Erhebung, die die Praxen des Schreibens und der Lektüre speziell in den genannten zwei Disziplinen fokussiert, wird dieser Frage nachgegangen. Der Beitrag stellt in einem ersten Schritt das Konzept und das methodische Vorgehen der Untersuchung vor (Frageborgen), und präsentiert in einem zweiten die ersten Resultate.

Schlagworte: Evaluation, Kursprogramme, Vorbereitung der Schriftlichkeit 


\title{
A Study in history and sciences high-school classes in Québéc
}

\begin{abstract}
In school reform discussions language has high importance. What is the importance of writing and reading in high-school? We conducted a study to describe how students practice reading and writing in history and science. We present the concept, the method including the questionnaires and first results on how highschool students report their writing-style in Québec.
\end{abstract}

Key words: pre-writing, course programme, evaluation

\section{Funzione epistemica dello scritto e discpline. Inchiesta nelle classisecondarie di storia e scienze del Québec}

\section{Riassunto}

Le riforme dei curricoli scolastici riconoscono alla lingua un ruolo fondamentale nella costruzione delle conoscenze da parte degli allievi. In questo contributo ci si pone la domanda circa il ruolo specifico dello scritto (ciò che si legge e si scrive) nell'insegnamento della storia delle scienze a livello di scuola secondaria. Per trovare una risposta, abbiamo condotto un'inchiesta sulla pratica di lettura e scrittura in queste discipline. Dopo aver presentato il quadro concettuale e la metodologia (questionario), esponiamo i primi risultati relativi al modo con cui gli allievi della scuola secondaria del Québec si rapportano allo scritto.

Parole chiave: preparazione alla scrittura, piani di formazione, valutazione 
